\title{
Childhood Metabolic Syndrome
}

\section{Must we define it to deal with it?}

RAM WEISS, MD, PHD

$\mathbf{T}$ he metabolic syndrome, also known as "syndrome X," describes a cluster of cardiovascular risk factors that have been shown to predict the development of cardiovascular disease and type 2 diabetes (1). Some suggest that the cluster is driven by the consequences of peripheral insulin resistance (2), whereas others believe that obesity-related inflammation is the culprit (3). Many putative molecular mechanisms can provide excellent explanations for both theories regarding the primary driving mechanism of the clustering of cardiovascular risk factors (4) and how each one accelerates atherogenesis (5). Yet teasing inflammation and peripheral insulin resistance apart in individual patients or even in large cohorts is difficult because of their coexistence and possible vicious exacerbation of each other. The general paradigm regarding the evolvement of the syndrome is that adipocyte dysfunction in some obese individuals who are genetically prone or exposed to specific environmental signals leads to peripheral insulin resistance and subclinical inflammation culminating in accelerated atherosclerosis and overt clinical manifestations (6). The clinical utility of defining this syndrome in children and adolescents has been debated, since some propose that from a clinical standpoint, addressing each component of the cluster individually has comparable clinical and predictive outcomes, whereas others suggest such definitions are inappropriate for the pediatric age group. This debate has important implications from an epidemiological and public health standpoint. Yet it is imperative to indicate that the underlying pathophysiology that leads to the typical metabolic milieu characteristic of individuals with obesity-driven peripheral insulin resistance and/or subclinical inflammation has common features in all ages and is postulated to be the "driving force" of the development of accelerated atherogenesis and altered glucose metabolism in susceptible individuals.

\section{COMPLEXITY OF DEFINITIONS OF THE METABOLIC SYNDROME}

IN CHILDHOOD_Several definitions of the metabolic syndrome in children have been proposed by various research groups $(7,8)$ and expert consensus (9), and the use of different definitions in the same patient cohort may result in different prevalence or prediction outcomes (10). All of the definitions share common features: First, all definitions include an obesity element (waist circumference or BMI), two "dyslipidemia" elements (elevated triglycerides and low HDL cholesterol), elevated blood pressure, and a component representing glucose metabolism (impaired fasting glucose or impaired glucose tolerance). All of these definitions are based on populationderived percentile thresholds for each component. Importantly, the choice of these elements in various definitions is in some cases a result of simplicity and cost (such as using a fasting blood sample) and the presence of good reference values (using BMI) and thus not necessarily the optimal choice one would have made for better diagnosis or prediction. Second, all

From the Department of Human Metabolism and Nutrition and the Department of Pediatrics, Hebrew University School of Medicine, Jerusalem, Israel.

Corresponding author: Ram Weiss, ram.weiss@ekmd.huji.ac.il.

This publication is based on the presentations at the 3rd World Congress on Controversies to Consensus in Diabetes, Obesity and Hypertension (CODHy). The Congress and the publication of this supplement were made possible in part by unrestricted educational grants from AstraZeneca, Boehringer Ingelheim, BristolMyers Squibb, Daiichi Sankyo, Eli Lilly, Ethicon Endo-Surgery, Generex Biotechnology, F. Hoffmann-La Roche, Janssen-Cilag, Johnson \& Johnson, Novo Nordisk, Medtronic, and Pfizer.

DOI: $10.2337 / \mathrm{dcll} 1-\mathrm{s} 214$

(C) 2011 by the American Diabetes Association. Readers may use this article as long as the work is properly cited, the use is educational and not for profit, and the work is not altered. See http://creativecommons.org/ licenses/by-nc-nd/3.0/ for details.

definitions use each component of the cluster as a dichotomous variable defined by a threshold and share the concept that all components have an equal "value" in the cumulative score. The latter two principles simplify the use of such definitions in everyday practice yet seem problematic in the sense that cardiovascular risk factors such as elevated fasting glucose and triglycerides or the degree of obesity represent continuous variables that signify risk, not necessarily in a linear fashion. Thus, for example, whereas increasing BMI during childhood represents a continuous risk factor for the development of coronary heart disease in adulthood even within the normal BMI range (11), severely obese children may have a significantly worse metabolic phenotype compared with moderately obese children (12). A seemingly "upper normal" fasting glucose in the context of obesity may signify future risk (13). Similarly, the fasting triglyceride level in late adolescence and its change within a brief follow-up of $\sim 5$ years can predict the development of diabetes and of coronary heart disease even when both measurements are below the threshold used in all of the proposed definitions. Thus, some have suggested that a "hypertriglyceridemic waist" phenotype can serve as a risk predictor for clinical purposes regardless of other components, highlighting a potential stronger metabolic impact of abdominal obesity and plasma triglyceride levels compared with other components of the definition (14). Although no such data exist in pediatric studies, it has been shown in adults that the presence of some combinations of components of the syndrome confers a worse prognosis than others. Specifically, over an 8-year follow-up, patients having a combination of central obesity, high blood pressure, and hyperglycemia had a 2.36-fold increase of incident cardiovascular events and a threefold increased risk of mortality (15). Thus, particular combinations of factors included in the definitions of the metabolic syndrome confer greater risks and raise questions regarding the equal value given in the overall score to each of the components.

Adding complexity to definitions of the metabolic syndrome is the problematic 
generalizability for populations of a different ethnicity. One of the conditions predicted by the presence of the metabolic syndrome is type 2 diabetes, a disease more common among children and adolescents from ethnic minorities in the U.S. (16) as well as in some European countries. Similarly, lipid values of African American children have a different distribution than Caucasians, which may lead to under-diagnosis based on the presently used thresholds (17). Individuals with a comparable BMI of different ethnic background (such as Asians compared with Caucasians) may have a significantly different body composition. Such differences translate into a greater percent body fat per given BMI, leading to an increased vulnerability to the adverse impact of increased specific body fat depots in individuals at lower BMI or waist circumference levels (18). In addition, ethnic background may have an effect on patterns of lipid partitioning in insulinresponsive tissues such as muscle and liver (19); thus, subjects with similar degrees of obesity may be entirely different in regards to their degree of peripheral insulin sensitivity. These observations imply that anthropometric and biochemical components used in definitions of the metabolic syndrome should be ethnicity specific and derived from outcome data of the relevant population (20). This result adds further complexity to the use of threshold-based definitions of the metabolic syndrome in childhood and emphasizes the difficulty of creating a "one fits all" definition for clinical practice.

The usefulness of metabolic syndrome definitions should be assessed in the context of their use, i.e., in the clinical setting or for research purposes. The clinical utility of such definitions in children has been questioned, and some advocate addressing individual risk factors in their clinical context instead of using a "syndromic" approach. For clinical purposes, an ideal definition should include components that are easily measured and represent a "stable" diagnosis, similar to other conditions and syndromes typically diagnosed in childhood. Moreover, a useful and clinically relevant definition should be able to reliably predict future clinical outcomes.

\section{STABILITY OF THE DIAGNOSIS OF METABOLIC SYNDROME IN CHILDHOOD-}

The stability of individual components of the syndrome from childhood to young adulthood has been shown to track "moderately well" with significant correlation coefficients of 0.4-0.6 for each component (21). Patterns of change in individual factors, defined as crossing the predefined thresholds between observations, have been shown to be more common in youth at risk (patients who a priori meet individual criteria) than in individuals at lower risk (22). Individual components of the syndrome have been shown to track from childhood to adulthood, emphasizing the importance of identifying abnormalities early in the life course (23). Tracking of the cluster during an 8-year follow-up has been shown to be stronger than the tracking of individual components, since the magnitude of the overall multiple risk index tracking correlation $(r=0.64)$ was significantly stronger than that noted for individual risk factors $(r=$ 0.34-0.57) (24). The diagnosis of the syndrome as a whole during a follow-up of 1-3 years during adolescence was shown to be relatively unstable (25), specifically when evaluated in population-derived cohorts. When tested in obese adolescents, i.e., those most prone to meet the criteria of the various definitions, the stability of the diagnosis was shown to be tightly associated with weight dynamics and changes in insulin sensitivity (26). Moreover, the persistence of the diagnosis in obese children over several visits was associated with accelerated fat gain, increased insulin response to oral glucose, and decreased insulin sensitivity and $\beta$-cell function, indicators of progressively greater risk for type 2 diabetes. Putative explanations for the "flexibility" of the diagnosis in adolescence in many studies may rely on the hormonal changes of puberty that induce a transient significant reduction of peripheral insulin sensitivity (27), the pubertal growth spurt that may result in significant body habitus changes and on the limited reproducibility and reliability of single assessments of blood pressure and glucose metabolism parameters in this age group (28). The fact that a single measurement of fasting glucose is used and a specific threshold chosen undermines the normal variability of up to $15 \mathrm{mg} / \mathrm{dL}$ observed in adults (29) may explain the crossing of the threshold in repeated measurements.

\section{PREDICTIVE VALUE OF THE METABOLIC SYNDROME IN} CHILDHOOD_-It has been shown using several longitudinal cohorts that meeting the criteria of the metabolic syndrome in childhood predicts the development of cardiovascular disease and type 2 diabetes in adulthood (30). Similarly, having specific components of the syndrome in childhood predicts the presence of "softer" outcomes such as left ventricular hypertrophy or increased intimal-medial thickness in childhood (31) and adulthood (32). Attempting to predict the presence of the syndrome itself in adulthood on the basis of meeting (or not meeting) its criteria in childhood shows fairly good specificity. This result translates to a good screening tool for ruling out future metabolic risk in individuals who do not meet the criteria in childhood (33). On the other hand, meeting the criteria in childhood shows a limited positive predictive value for the presence of the syndrome in adulthood (34). The combination of metabolic risk factors can increase the probability that individuals with a positive test are truly diseased in adulthood. The presence of obesity in childhood seems to be the strongest predictor of the presence of the syndrome in adulthood (35), again suggesting that for clinical or prediction purposes (some elements of the syndrome may carry more weight than others). Additional elements of the pediatric history taking, that are not included in traditional metabolic syndrome definitions, can significantly improve the predictive value of having the syndrome in adulthood. Such elements include a positive family history for type 2 diabetes or cardiovascular disease (36), low birth weight and early catch-up growth (37), "early versus late" growth and maturation patterns (38), socioeconomic status in childhood (39), sedentary behavior (40), and specific dietary constituents. Although no such data have been published from pediatric cohorts, it is reasonable to assume that, as in adults, the ability of the metabolic syndrome to predict incident cardiovascular disease or diabetes depends on the definition used and on the population studied (41).

\section{SHOULD OTHER COMPONENTS BE INCLUDED IN THE DEFINITION OF THE METABOLIC SYNDROME IN CHILDHOOD? - The metabolic phe-} notype of obese children who meet the definitions of the metabolic syndrome is variable, yet some clinical and biochemical associations are typically observed. 
Body fat distribution has a critical role in the determination of whole-body insulin sensitivity and its consequences. The relation of obesity and peripheral insulin resistance depends more on the lipid distribution (or "lipid partitioning") in specific fat depots rather than on the absolute amount of fat per se. Importantly, these distinctions are not reflected in BMI assessments. Different lipid depots have distinct metabolic characteristics that are reflected by their adipocytokine and cytokine secretion profile, sensitivity to hormones typically affecting adipose tissue (such as norepinephrine or insulin), and anatomical blood supply and drainage (portal vs. systemic) (42). The secretory role of visceral fat-derived proinflammatory cytokines and adipocytokines (such as adiponectin [43] and leptin) appears to be directly associated with obesity and insulin resistance. Indeed, increased visceral fat accumulation in obese children has been associated with increased insulin resistance and with cardiovascular risk factor clustering as well as with worsening of each factor individually (44). Some obese children tend to demonstrate a lipid-partitioning pattern characterized by a large visceral fat depot along with a relatively smaller subcutaneous fat depot. This lipid-partitioning profile is associated with an adverse metabolic profile in comparison with individuals with larger subcutaneous fat depots, even when the latter have greater BMI and percent body fat and may thus be seemingly "more obese" (45). Waist circumference has been demonstrated to be an independent predictor of insulin resistance and intra-abdominal fat independent of BMI in obese adolescents (46). Moreover, waist circumference has been shown to be tightly linked to systolic and diastolic blood pressure and to triglyceride and HDL cholesterol concentrations in this age group (47). For these reasons, the International Diabetes Federation task force (9) chose waist circumference, the best anthropometric correlate of intra-abdominal fat, as the "obesity factor" of the pediatric metabolic syndrome definition.

Lipid deposition in muscle and liver represents another determinant of the sensitivity of these tissues to the metabolic effects of insulin. Intramyocellular lipid deposition is inversely correlated with peripheral insulin sensitivity and has been demonstrated to be increased in offspring of type 2 diabetic patients and in obese children with impaired glucose tolerance (48). Importantly, the association of intramyocellular fat and insulin sensitivity is further determined by the size of lipid droplets and probably their localization within the myocyte. (These two factors explain the paradox of the presence of increased intramyocellular fat in endurance athletes who have comparable intramuscular fat to obese patients with diabetes. The difference lies in the smaller size of their lipid droplets, probably making them more accessible to oxidation.) Similarly, hepatic fat accumulation is strongly associated with obesity and with hepatic resistance to the action of insulin in the context of pathways related to glucose metabolism and is also associated with an adverse cardiovascular risk profile in children (49). Because both tissues develop insulin resistance in association with increased lipid deposition, the normal adaptive response consists of increased insulin secretion along with reduced insulin clearance, leading to increased circulating insulin levels (hyperinsulinemia). Importantly, other metabolic pathways within the liver that are not involved in glucose metabolism or other insulin-sensitive tissues that do not share the pattern of increased lipid deposition within them, such as the kidney or the ovary, maintain their baseline insulin sensitivity levels yet are now exposed to hyperinsulinemia. This occurrence may result in a normal response of these tissues to elevated insulin levels and manifest as sodium retention and reduced uric acid clearance by the kidney (50) (potentially elevating systemic blood pressure) and by increased androgen production by the theca cells of the ovary manifesting as polycystic ovary syndrome (51). Other metabolic pathways within the liver, specifically those related to lipoprotein metabolism, maintain their baseline insulin sensitivity (unlike those pathways related to glucose metabolism) and respond to the elevated insulin levels in a pattern that creates the typical dyslipidemia characteristic of insulin-resistant individuals. This result is manifested as elevated concentrations of large VLDL particles, low HDL cholesterol, and elevated small dense LDL particle concentration (52). Some suggest that hepatic deposition of lipid is not a primary process but a "normal" response to elevated circulating insulin levels induced by muscle insulin resistance and thus that hepatic steatosis, typically found in obese adolescents with the metabolic syndrome, is the result and not one of the culprits of the adverse metabolic phenotype characteristic of insulin-resistant individuals. Hyperinsulinemia may additionally induce an activation of the sympathetic nervous system and affect the metabolism and secretion of proinflammatory cytokines as well as coagulation mediators (53), as reflected by the elevated concentrations of such cytokines commonly observed in obese insulin-resistant individuals.

Inflammatory mediators have been suggested to be the primary insult leading to the development of insulin resistance and future atherogenesis in patients with the metabolic syndrome. An association of C-reactive protein with adiposity, fasting insulin, dyslipidemia, and blood pressure has been shown in prepubertal children. In healthy adolescents, C-reactive protein was significantly associated with indices of insulin resistance and components of the syndrome, yet this association was attenuated after adjustment for degree of adiposity, suggesting that obesity possibly precedes the appearance of biochemical markers of enhanced inflammation in the development of cardiovascular risk factors in childhood. Similarly, interleukin-6 and tumor necrosis factor- $\alpha$ have been shown to be increased in adolescents with the metabolic syndrome, reflecting the subclinical inflammatory process that is activated in these individuals. Thus, reduced levels of adiponectin and increased inflammatory cytokines seem to be nontraditional factors accompanying the classic components of the syndrome. Early markers of atherogenesis such as endothelial dysfunction have been associated with the presence of the metabolic syndrome in adults, and a small number of reports show comparable findings in children manifesting the syndrome (54).

Several groups have performed a factor analysis of components of the metabolic syndrome in cohorts of adults and children $(55,56)$ to reveal associations observed between its components. These studies revealed that obesity and its related peripheral insulin resistance seem to cluster with the majority of the traditional components of the syndrome, yet also cluster with other factors, such as increased fibrinolysis, endothelial dysfunction, and subclinical inflammation, which seem to be part of the typical metabolic milieu of the insulin-resistant individual yet are not routinely assessed or used for clinical decision-making or for 
the sake of risk stratification. Figure 1 demonstrates the classic components used in definitions of the metabolic syndrome in childhood, regardless of specific thresholds, along with other historyderived, anthropometric, and biochemical parameters that are associated with its presence. While the utility of such parameters for diagnostic or treatment purposes is still unknown, the caregiver is advised to seek the ones he can easily obtain to be able to address them (such as ovarian-derived hyperandrogenism) or at least be aware of their presence and significance. Additional conditions typically found in obese adolescents who meet criteria of the syndrome are also shown to raise the index of suspicion for their presence.

\section{CLINICAL UTILITY OF METABOLIC SYNDROME DEFINITIONS IN CHILDHOOD}

The observations reviewed in this article highlight the importance of primary and secondary prevention of the progression of early cardiovascular risk factors in children and adolescents. Identification of the children who are at high a priori risk to develop the syndrome, based on a thorough family history and objective data related to pregnancy, labor, and the postnatal period, can be performed by any caregiver. Because components of the metabolic syndrome tend to track from childhood to adulthood, primary prevention of their development or early reversal of their presence in childhood are of paramount importance. Interventions such as early implementation of appropriate dietary and lifestyle practices aimed at primary prevention should be suggested during or even before pregnancy. Diet-induced weight loss and bariatric surgery have been attempted in obese children with the presence of cardiovascular risk factors or overt disease such as type 2 diabetes. Such interventions have shown that the level of cardiovascular risk factors related to the metabolic syndrome can be reduced (57) and that the presence of type 2 diabetes can be eliminated. Because these interventions are expensive, labor intensive, and potentially invasive and nonreversible in nature, selection of those obese youth who may benefit most from them is crucial. Measures such as risk factor clustering (metabolic

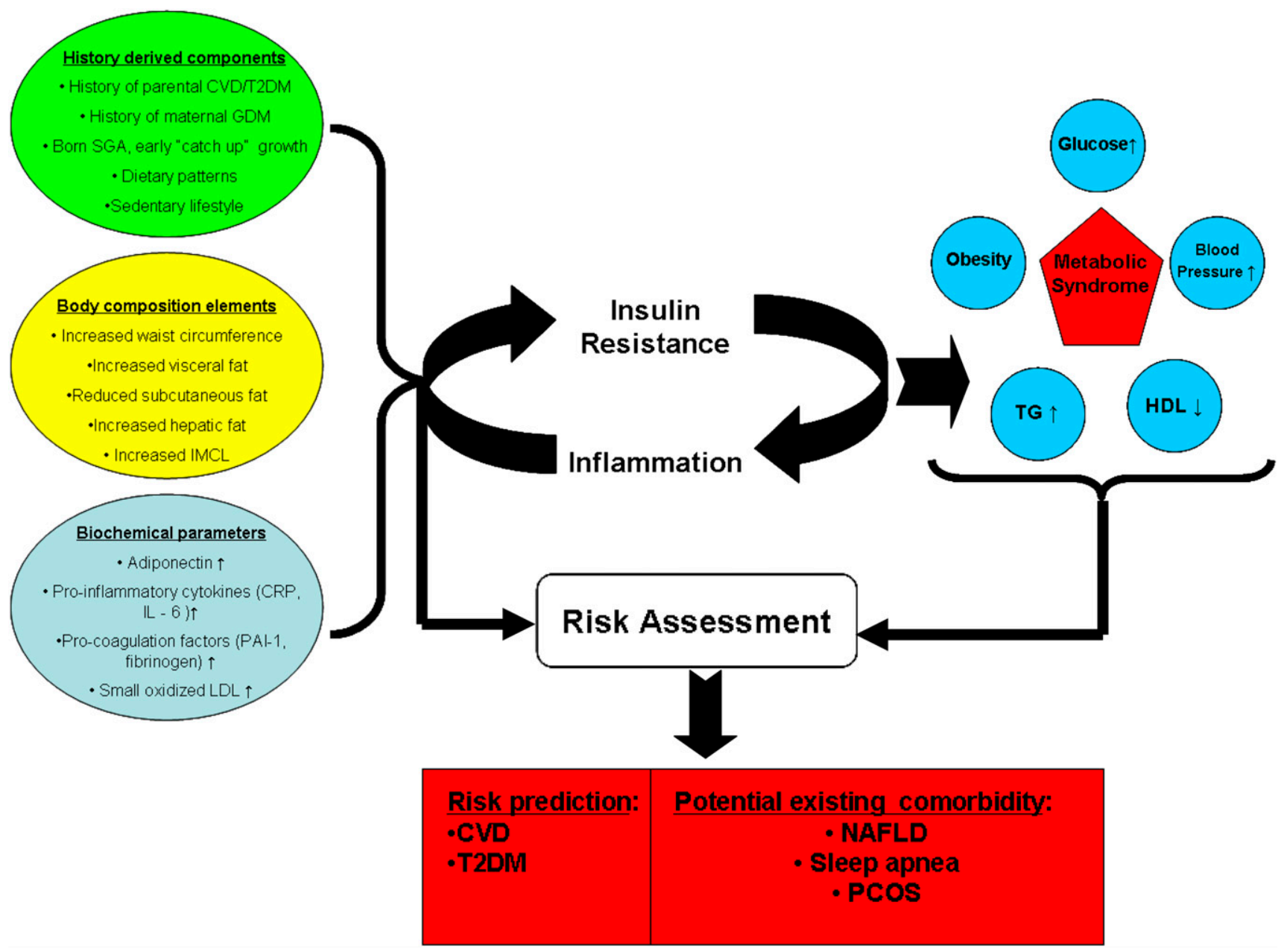

Figure 1-Several genetic, environmental, lipid partitioning, and biochemical factors, shown on the left, promote the development of subclinical inflammation and insulin resistance, both of which can exacerbate each other. This is the postulated driving mechanism that leads to the development of classic components of the metabolic syndrome, shown on the right. Both the classic definitions and the addition of the predisposing and promoting factors can contribute to risk assessment of the individual patients in regard to existing comorbidities and to future cardiovascular and diabetes risk. CRP, C-reactive peptide; CVD, cardiovascular disease; GDM, gestational diabetes mellitus; IL-6, interleukin-6; IMCL, intramyocellular lipid; NAFLD, nonalcoholic fatty liver disease; PAI-1, plasminogen activator inhibitor 1; PCOS, polycystic ovarian syndrome; SGA, small for gestational age; T2DM, type 2 diabetes; TG, triglycerides. 
syndrome definitions) and its dynamics over time can serve as selection and followup tools for the assessment of such interventions.

CONCLUSIONS_Clustering of cardiovascular risk factors, the development of which is driven by adipocyte dysfunction leading to subclinical inflammation and peripheral insulin resistance, is present in children and adults. Such clustering may be associated with specific morbidity in childhood and also predicts the presence of adverse outcomes in adulthood. Obesity per se in a child does not necessarily mean that the syndrome is present. The pattern of lipid partitioning, adipocytokine and cytokine profile, and presence of genetically determined factors (such as ethnicity, family history of type 2 diabetes, and others) is crucial for the development of the adverse metabolic phenotype typical of individuals who develop the syndrome. Definitions of the syndrome that are based on thresholds, despite being controversial and difficult to generalize for diverse populations, may be useful in clinical practice for the identification and followup of those youth who may benefit most from therapeutic interventions. Importantly, the components used to diagnose the syndrome represent a continuum of risk and should thus be addressed and followed even when they are seemingly "normal." This continuum is not necessarily linear, and specific components can confer entirely different risk despite being classified as "normal" or "abnormal" when assessed strictly by using a single threshold value. The caregiver is advised to seek comorbid conditions and carefully follow patients who meet the criteria in childhood, since most of the conditions do not manifest as overt disease and can potentially be addressed early in their development. Despite the presence of multiple definitions and the difficulty of using them in different populations, the general pathophysiological processes are similar across age and ethnicity. Thus, the caregiver should use prudent clinical judgment to address the typical phenotype associated with the metabolic syndrome in children and adolescents and adhere to the simple wisdom of, "When I see a bird that walks like a duck and swims like a duck and quacks like a duck, I call that bird a duck" (James Whitcomb Riley, "The Hoosier Poet", 1883).
Acknowledgments-No potential conflicts of interest relevant to this article were reported.

\section{References}

1. Cornier MA, Dabelea D, Hernandez TL, et al. The metabolic syndrome. Endocr Rev 2008;29:777-822

2. Reaven GM. Banting lecture 1988. Role of insulin resistance in human disease. Diabetes 1988:37:1595-1607

3. Yudkin JS. Insulin resistance and the metabolic syndrome-or the pitfalls of epidemiology. Diabetologia 2007;50:15761586

4. de Luca C, Olefsky JM. Inflammation and insulin resistance. FEBS Lett 2008;582: 97-105

5. Fonseca VA. The effects of insulin on the endothelium. Endocrinol Metab Clin North Am 2007;36(Suppl. 2):20-26

6. Guilherme A, Virbasius JV, Puri V, Czech MP. Adipocyte dysfunctions linking obesity to insulin resistance and type 2 diabetes. Nat Rev Mol Cell Biol 2008;9: 367-377

7. de Ferranti SD, Gauvreau K, Ludwig DS, Neufeld EJ, Newburger JW, Rifai N. Prevalence of the metabolic syndrome in American adolescents: findings from the Third National Health and Nutrition Examination Survey. Circulation 2004;110: 2494-2497

8. Weiss R, Dziura J, Burgert TS, et al. Obesity and the metabolic syndrome in children and adolescents. N Engl J Med 2004; 350:2362-2374

9. Zimmet P, Alberti G, Kaufman F, et al. The metabolic syndrome in children and adolescents. Lancet 2007;369:20592061

10. Lee S, Bacha F, Gungor N, Arslanian S. Comparison of different definitions of pediatric metabolic syndrome: relation to abdominal adiposity, insulin resistance, adiponectin, and inflammatory biomarkers. J Pediatr 2008;152:177-184

11. Baker JL, Olsen LW, Sørensen TI. Childhood body-mass index and the risk of coronary heart disease in adulthood. N Engl J Med 2007;357:2329-2337

12. Freedman DS, Mei Z, Srinivasan SR, Berenson GS, Dietz WH. Cardiovascular risk factors and excess adiposity among overweight children and adolescents: the Bogalusa Heart Study. J Pediatr 2007;150: 12-17

13. Tirosh A, Shai I, Tekes-Manova D, et al. Normal fasting plasma glucose levels and type 2 diabetes in young men. N Engl J Med 2005;353:1454-1462

14. Esmaillzadeh A, Mirmiran P, Azizi F. Clustering of metabolic abnormalities in adolescents with the hypertriglyceridemic waist phenotype. Am J Clin Nutr 2006;83: $36-46$
15. Franco OH, Massaro JM, Civil J, Cobain MR, O'Malley B, D'Agostino RB Sr. Trajectories of entering the metabolic syndrome: the Framingham Heart Study. Circulation 2009;120:1943-1950

16. Dabelea D, Pettitt DJ, Jones KL, Arslanian SA. Type 2 diabetes mellitus in minority children and adolescents: an emerging problem. Endocrinol Metab Clin North Am 1999;28:709-729

17. Johnson WD, Kroon JJ, Greenway FL, Bouchard C, Ryan D, Katzmarzyk PT. Prevalence of risk factors for metabolic syndrome in adolescents: National Health and Nutrition Examination Survey (NHANES), 2001-2006. Arch Pediatr Adolesc Med 2009;163:371-377

18. Deurenberg-Yap M, Chew SK, Deurenberg $\mathrm{P}$. Elevated body fat percentage and cardiovascular risks at low body mass index levels among Singaporean Chinese, Malays and Indians. Obes Rev 2002;3:209-215

19. Liska D, Dufour S, Zern TL, et al. Interethnic differences in muscle, liver and abdominal fat partitioning in obese adolescents. PLoS ONE 2007;2:e569

20. Misra A, Wasir JS, Vikram NK. Waist circumference criteria for the diagnosis of abdominal obesity are not applicable uniformly to all populations and ethnic groups. Nutrition 2005;21:969-976

21. Katzmarzyk PT, Pérusse L, Malina RM, Bergeron J, Després JP, Bouchard C. Stability of indicators of the metabolic syndrome from childhood and adolescence to young adulthood: the Québec Family Study. J Clin Epidemiol 2001;54:190195

22. Li C, Ford ES, Huang TT, Sun SS, Goodman E. Patterns of change in cardiometabolic risk factors associated with the metabolic syndrome among children and adolescents: the Fels Longitudinal Study. J Pediatr 2009;155:S5.e9-e16

23. Webber LS, Srinivasan SR, Wattigney WA, Berenson GS. Tracking of serum lipids and lipoproteins from childhood to adulthood: the Bogalusa Heart Study. Am J Epidemiol 1991;133:884-899

24. Bao W, Srinivasan SR, Wattigney WA, Berenson GS. Persistence of multiple cardiovascular risk clustering related to syndrome $\mathrm{X}$ from childhood to young adulthood: the Bogalusa Heart Study. Arch Intern Med 1994;154:1842-1847

25. Gustafson JK, Yanoff LB, Easter BD, et al. The stability of metabolic syndrome in children and adolescents. J Clin Endocrinol Metab 2009;94:4828-4834

26. Weiss R, Shaw M, Savoye M, Caprio S. Obesity dynamics and cardiovascular risk factor stability in obese adolescents. Pediatr Diabetes 2009; 10:360-367

27. Goran MI, Gower BA. Longitudinal study on pubertal insulin resistance. Diabetes 2001;50:2444-2450

28. Libman IM, Barinas-Mitchell E, Bartucci A, Robertson R, Arslanian S. Reproducibility 
of the oral glucose tolerance test in overweight children. J Clin Endocrinol Metab 2008;93:4231-4237

29. Mooy JM, Grootenhuis PA, de Vries H, et al. Intra-individual variation of glucose, specific insulin and proinsulin concentrations measured by two oral glucose tolerance tests in a general Caucasian population: the Hoorn Study. Diabetologia 1996;39:298-305

30. Franks PW, Hanson RL, Knowler WC, et al. Childhood predictors of youngonset type 2 diabetes. Diabetes 2007;56: 2964-2972

31. Reinehr T, Wunsch R, de Sousa G, Toschke AM. Relationship between metabolic syndrome definitions for children and adolescents and intima-media thickness. Atherosclerosis 2008;199:193-200

32. Juonala M, Viikari JS, Rönnemaa T, et al. Associations of dyslipidemias from childhood to adulthood with carotid intimamedia thickness, elasticity, and brachial flow-mediated dilatation in adulthood: the Cardiovascular Risk in Young Finns Study. Arterioscler Thromb Vasc Biol 2008;28: 1012-1017

33. Chen W, Srinivasan SR, Li S, Xu J, Berenson GS. Metabolic syndrome variables at low levels in childhood are beneficially associated with adulthood cardiovascular risk: the Bogalusa Heart Study. Diabetes Care 2005;28:126-131

34. Schubert CM, Sun SS, Burns TL, Morrison JA, Huang TT. Predictive ability of childhood metabolic components for adult metabolic syndrome and type 2 diabetes. Pediatr J 2009; 155:S6.el-e7

35. Burns TL, Letuchy EM, Paulos R, Witt J. Childhood predictors of the metabolic syndrome in middle-aged adults: the Muscatine study. J Pediatr 2009;155:S5.e17-e26

36. Bao W, Srinivasan SR, Wattigney WA, Berenson GS. The relation of parental cardiovascular disease to risk factors in children and young adults: The Bogalusa Heart Study. Circulation 1995;91:365-371

37. Lévy-Marchal C, Czernichow P. Small for gestational age and the metabolic syndrome: which mechanism is suggested by epidemiological and clinical studies? Horm Res 2006;65(Suppl. 3):123-130

38. Sun SS, Schubert CM. Prolonged juvenile states and delay of cardiovascular and metabolic risk factors: the Fels Longitudinal study. J Pediatr 2009;155:S7.el-e6

39. Kivimäki M, Smith GD, Juonala M, et al. Socioeconomic position in childhood and adult cardiovascular risk factors, vascular structure, and function: cardiovascular risk in young Finns study. Heart 2006;92: 474-480

40. Ekelund U, Brage S, Froberg K, et al. TV viewing and physical activity are independently associated with metabolic risk in children: the European Youth Heart Study. PLoS Med 2006;3:e488

41. Gami AS, Witt BJ, Howard DE, et al. Metabolic syndrome and risk of incident cardiovascular events and death: a systematic review and meta-analysis of longitudinal studies. J Am Coll Cardiol 2007; 49:403-414

42. Wajchenberg BL. Subcutaneous and visceral adipose tissue: their relation to the metabolic syndrome. Endocr Rev 2000; 21:697-738

43. Shaibi GQ, Cruz ML, Weigensberg MJ, et al. Adiponectin independently predicts metabolic syndrome in overweight Latino youth. J Clin Endocrinol Metab 2007;92: 1809-1813

44. Bacha F, Saad R, Gungor N, Janosky J, Arslanian SA. Obesity, regional fat distribution, and syndrome $\mathrm{X}$ in obese black versus white adolescents: race differential in diabetogenic and atherogenic risk factors. J Clin Endocrinol Metab 2003;88: 2534-2540

45. Taksali SE, Caprio S, Dziura J, et al. High visceral and low abdominal subcutaneous fat stores in the obese adolescent: a determinant of an adverse metabolic phenotype. Diabetes 2008;57:367-371

46. Lee S, Bacha F, Gungor N, Arslanian SA. Waist circumference is an independent predictor of insulin resistance in black and white youths. J Pediatr 2006;148:188-194

47. Lee S, Bacha F, Arslanian SA. Waist circumference, blood pressure, and lipid components of the metabolic syndrome. J Pediatr 2006;149:809-816

48. Weiss R, Dufour S, Taksali SE, et al. Prediabetes in obese youth: a syndrome of impaired glucose tolerance, severe insulin resistance, and altered myocellular and abdominal fat partitioning. Lancet 2003; 362:951-957

49. Cali AM, De Oliveira AM, Kim H, et al. Glucose dysregulation and hepatic steatosis in obese adolescents: is there a link? Hepatology 2009;49:1896-1903

50. Facchini F, Chen YD, Hollenbeck CB, Reaven GM. Relationship between resistance to insulin-mediated glucose uptake, urinary uric acid clearance, and plasma uric acid concentration. JAMA 1991;266:3008-3011

51. Dunaif A. Insulin resistance and the polycystic ovary syndrome: mechanism and implications for pathogenesis. Endocr Rev 1997;18:774-800

52. Krauss RM, Siri PW. Metabolic abnormalities: triglyceride and low-density lipoprotein. Endocrinol Metab Clin North Am 2004:33:405-415

53. Van Gaal LF, Mertens IL, De Block CE. Mechanisms linking obesity with cardiovascular disease. Nature 2006:444:875880

54. Lee S, Gungor N, Bacha F, Arslanian S. Insulin resistance: link to the components of the metabolic syndrome and biomarkers of endothelial dysfunction in youth. Diabetes Care 2007;30:2091-2097

55. Li C, Ford ES. Is there a single underlying factor for the metabolic syndrome in adolescents? A confirmatory factor analysis. Diabetes Care 2007;30:1556-1561

56. Goodman E, Dolan LM, Morrison JA, Daniels SR. Factor analysis of clustered cardiovascular risks in adolescence: obesity is the predominant correlate of risk among youth. Circulation 2005;111:19701977

57. Savoye M, Shaw M, Dziura J, et al. Effects of a weight management program on body composition and metabolic parameters in overweight children: a randomized controlled trial. JAMA 2007;297:2697-2704 